\title{
Scanning thermal ferromagnetic resonance microscopy of Fe-GaAs heterostructures and Ni-nanodots
}

\author{
R. Meckenstock, D. Spoddig and J. Pelzl \\ Institut fuer Experimentalphysik 3 AG, Ruhr-Universitaet, Bochum, D-44801 Germany
}

Due to the trend of miniaturisation the control of magnetic and electric properties on micrometer and nanometer scales has become increasingly important for the performance of modern magneto-electronic devices. Ferromagnetic resonance (FMR) provides a very sensitive tool for the study of magnetic properties of magnetically ordered materials. The conventional FMR, however, suffers from the lack local resolution particularly when a cavity is used. A promising means to overcome this problem offers the local detection of the FMR by photothermal methods [1]. With the photothermally modulated (PM-) FMR a lateral resolutions of about $1 \mu \mathrm{m}$ can be achieved at a high sensitivity. PM-FMR relies on local periodic heating of the sample by an intensity modulated laser beam [2]. Applying this technique to magnetic film semiconductor heterostructures we were able to image simultaneously magnetic properties of the ferromagnetic film and the electric properties of the semiconductor substrate. Very recently we also succeeded to extend the resolution of the FMR for the first time into nanometer scale by applying STM near field technique. This new method STM-FMR relies on the detection of the thermo-elastic response due to the dissipated energy at ferromagnetic resonance.

The experimental setup for both techniques is based on a microwave cavity with a conventional FMR spectrometer working in X-band. The sample is placed on the bottom plate of the cavity to allow access for the STM or the focussed laser beam respectively. In this work we report on first STM-FMR results obtained from a Ni-film with nano-dots and PM-FMR measurements on patterned Fe-GaAs heterostructure. The nano structured Ni-film was electrolytically grown on (111) Au on a mica substrate. The average thickness of the Nifilm was about $120 \mathrm{~nm}$. AFM and x-ray analysis of the Ni-film show (111) texture and column like growth leading to a distinct nano-dot structure (FIG. 1a). The shape distribution of the dots has a maximum of 150 $\mathrm{nm}$ at height and at width. For the FMR measurements the STM probe was positioned on top of a dot. Passing through the resonance by changing the external magnetic field the control voltage of the z-piezo was varied to maintain the tunnelling current constant. The variation of the control voltage as a function of the magnetic field is a measure of the thermo-elastic expansion of the dot due to the heating of the dissipated microwave power in resonance. The line positions of resonance signals measured from a slim and broad dot (FIG. 1b) are shifted by about $30 \mathrm{mT}$ due to the shape anisotropy. The FMR line width obtained from a single dot is close to the intrinsic line width deduced from the frequency dependent conventional FMR. In summary we believe that the presented results represent the first real FMR measurement of a magnetic nano-dot. Figure 2 displays the results of the PM-FMR from a Fe-GaAs heterostructure. This sample has a laterally structured 7nm (001)Fe film shown in the microscope picture (FIG. 2i) and a high electron mobility transistor (HEMT-) structure in the GaAs, which is located in a vertical stripe on the right site of the sample (low intensity area in FIG. 2ii). The low intensity is explained by the lower number of photo carriers induced by the laser light due to the non conducting layer of the HEMT-structure. Proceeding from the result of the PM-FMR (FIG. 2iii) and complementary angle dependent FMR measurements we found that the surface anisotropy of the Fe-mesa deposited on the HEMT-structure (b) is decreased by a factor of 2 compared to the Fe-mesa directly on GaAs (a). The uniaxial in-plane anisotropy was slightly decreased by about $10 \%$ between positions $b$ and $a$. References

[1] A. Mandelis, Progress in Photothermal and Photoacoustic Science and Technology, Vol.2., PTR Prentice Hall, Englwood Cliffs, 1994.

[2] R. Meckenstock, O. von Geisau, J. Pelzl and J.A. Wolf, J. Apl. Phys. 77 (1995) 6439. 
[3] This research was supported by the Sonderforschungsbereich 491 and the Graduiertenkolleg 384. The aid of Prof. Jean Pierre Bucher of IPCMS Strassbourg is gratefully acknowledged.
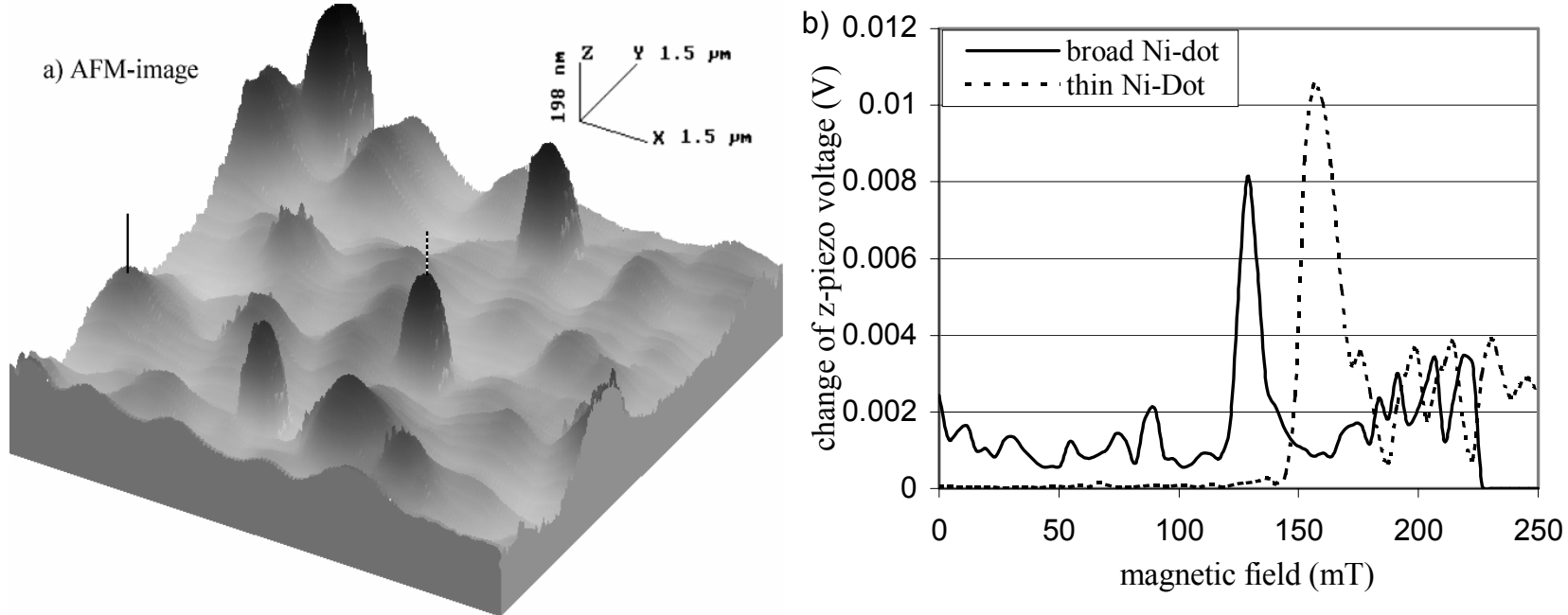

FIG. 1. a) AFM image of the Ni dots. b) Modulated thermo-elastic microwave resonance from the two indicated dots.
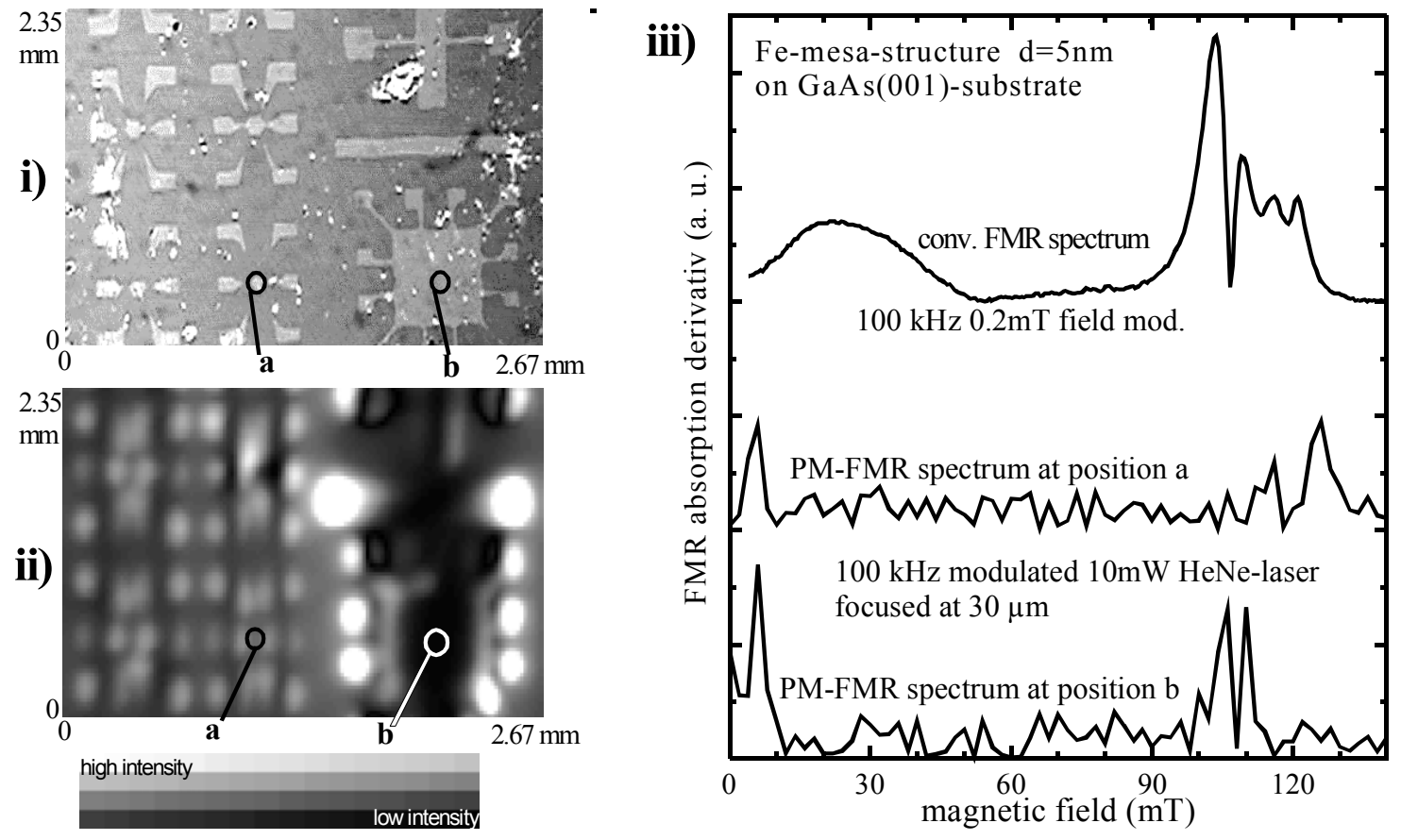

FIG. 2. i) Optical microscope image of structured Fe surface. ii) Greyscale image of the field independent PM-FMR response from the GaAs substrate. iii) Microwave response versus external magnetic field in [110] direction. Integral conventional FMR-spectrum, PM-FMR-spectrum of Fe-mesa on pure GaAs (position a), PM-FMR-spectrum of Fe-mesa with HEMT below (position b) 